\title{
A Social Media Organizational Productivity Model: Insights From Public Health Professionals
}

Hamad Ghaleb Dailah $\mathrm{Sr}^{1}$, PhD; Muhammad Naeem ${ }^{2}$, DBA

${ }^{1}$ Faculty of Nursing, Jazan University, Jazan, Saudi Arabia

${ }^{2}$ University of Gloucestershire, Cheltenham, United Kingdom

\section{Corresponding Author:}

Hamad Ghaleb Dailah Sr, PhD

Faculty of Nursing

Jazan University

Al Maarefah Rd

Jazan

Saudi Arabia

Phone: 966173295000

Email: drhamaddilah@gmail.com

\section{Abstract}

Background: Many previous studies have explored socialization-oriented social media (SM), but their reach has been limited to the context of information exchange for common personal interests. This study focuses on work-oriented SM, which can enhance organizational networking and productivity levels in the context of public hospitals.

Objective: This study aims to provide a theoretical framework to explain how the use of SM can enhance the skills of health professionals and levels of organizational productivity in uncertain environments.

Methods: A total of 2 distinct forms of data collection techniques were combined: focus groups and semistructured interviews. Both were conducted with doctors and nurses in Saudi public sector hospitals.

Results: The findings reveal that the use of SM can create professional socialization at the level of the institution, and this can enhance skills, knowledge, decision making, and the overall level of organizational productivity. The increasing use of SM creates collaboration between health experts (particularly endocrinologists and pulmonologists in this case) who arrange video calls to share best practices in terms of medication, diet, and health care plans for patients with multiple diseases. Many of these patients are particularly vulnerable, given the wider context of the current global pandemic.

Conclusions: This study culminates in the Social Media Organizational Productivity model, which provides insights into how SM has increased the accessibility of health professionals through the use of technology. Access to such professionals creates a patient-centric approach and a culture of shared communication for dealing with high-risk patients during the current global pandemic.

(J Med Internet Res 2021;23(5):e23792) doi: 10.2196/23792

\section{KEYWORDS}

social media; professional socialization; uncertainty; institutional theory; motivation; public hospital; health professionals

\section{Introduction}

\section{Background}

The flexible, agile, collaborative, informal, unstructured, and spontaneous nature of social media (SM) platforms has given rise to more and better social interactions, social support, knowledge sharing, and social expectations [1,2]. There are various kinds of SM, such as personal platforms (ie, Skype and WhatsApp) and organizational SM platforms (ie, Microsoft Yammer). Thus, SM is heterogeneous at both the personal and organizational levels. However, contemporary employees cannot differentiate between personal and corporate SM. On the basis of this heterogeneity and motivated by the influence of the SM world, workplaces have begun to embrace SM for socialization and work-oriented purposes [3,4]. Work-oriented SM types include web-based platforms that can be used in the workplace to facilitate collaboration. These forms of SM can also generate resources and facilitate the sharing of work-related content and information. They can support internal organizational communication and can be used to track events and 
workstreams. They are also reliable forms of support for task management [5,6]. However, socialization-oriented SM includes web-based platforms that facilitate the sharing of personal and social information and that enable people to develop expressive links that can shape identities through normative expectations and emotional and social forms of support $[6,7]$.

Existing literature on SM has tended to focus on exploring socialization-oriented SM, which is limited to information exchange for personal common interests. However, this study focuses on work-oriented SM that can influence organizational productivity in the setting of Saudi public sector hospitals. SM plays a very important role in supporting communication among employees in organizations [8]. A key previous study highlighted the importance of relationships and social interactions to achieve common objectives [8]. Virtuality theory advocates the use of SM if it is required to alter patterns of social exchange ties. However, it overlooks the consequences of such social exchange ties in organizational settings. Previous studies have measured the impact of patient tweets on hospital quality improvements, but these studies failed to produce the expected results $[6,9,10]$. Approximately $60 \%$ of physicians use SM to monitor patient health and education. Furthermore, these physicians use SM sites to inform patient drug adherence and behavioral changes that can enhance the level of patient compliance and positive outcomes $[6,9,10]$. Conversely, a survey of 480 physicians revealed that $68 \%$ of physicians hesitate to use SM sites for patient, personal, and professional connections. However, there is little research on how the use of SM for communication purposes influences organizational productivity in the Saudi health sector setting. High-quality SM relationships make a significant contribution to employee performance, but there is little understanding of this contribution in public hospitals in Saudi Arabia [11].

There is evidence of the popularity of SM among Saudi men and women, as approximately $41 \%$ of the Saudi population actively uses various SM platforms [2]. According to recent statistics, some 9.9 million Saudi Arabians regularly use Twitter. Saudi Arabians are the fourth highest users of Twitter in the world [12]. Facebook, WhatsApp, and Twitter are used to exchange audio, videos, text, views, and information and to meet personal and organizational interests. The Saudi government has restricted the use of some SM platforms because of social campaigns by SM activists between 2013 and 2017 [13]. However, after this time, the Saudi government lifted the ban and allowed the use of the SM platforms [13]. Public hospitals in Saudi Arabia are under the control of the Saudi Ministry of Health. It is therefore interesting to analyze the specific rules, regulations, policies, hierarchal structure, and power uses that can influence the use of SM platforms, even for productive purposes in Saudi public sector hospitals.

Although an ample body of literature has explored the effective use of SM at individual levels as well as in the organizational communication context in Western culture [14,15], there is evidence that Arab public organizational culture is unique and different from Western public organizational culture. This might be because of some inherent cultural attributes, such as authoritative management styles, hierarchal structures, a preference for top-down communication, low staff empowerment, and strong social networking among those with sufficient resources. Work overload and favoritism might also be factors. These are some of the unique features of Saudi public sector organizations that strongly influence the use of SM platforms at both the individual and organizational levels. There is little understanding of how public hospitals in Arab countries such as Saudi Arabia manage SM use. This study introduces a theoretical framework to guide how SM can drive productivity in Saudi public sector hospitals.

\section{Literature Review}

Deep penetration of SM has been observed in many workplaces. Nowadays, there is an increasing trend among companies to strategically implement such tools to improve their organizational activities and support their workforces [16]. Leading organizations regularly use personal, public, and popular SM such as LinkedIn, Twitter, and Facebook to improve employee engagement, customer service, talent recruitment, marketing, and knowledge sharing [17,18]. Research has described SM use in health information management as "the activities that people perform to acquire, organize, maintain, share, retrieve, and use health information items to complete healthcare tasks and fulfil their needs" [19]. The increasing use of SM in the workplace has led to the emergence of various socially connected software that companies commonly use to pursue their business activities and goals [5]. In addition, where the aim is to support employees, such use is considered promising for excellent organizational performance $[9,20]$.

In addition to extending access to public and general SM platforms such as Twitter and Facebook in workplaces [10,20], more emphasis is being placed on developing and implementing professional and more specific SM technologies. Examples include IBM connections, Slack, Jive, Facebook Workplace, DingTalk, and Microsoft Yammer. Consequently, it has become possible for different types of SM platforms to coexist in organizations. These can be used by workers for work and/or during work time for information exchange and effectiveness in workplaces. However, companies and executives may sometimes perceive SM as controversial [16]. This begs the question: How does the utilization of different kinds of SM platforms in the workplace influence organizational productivity, and what can be learned specifically from public sector hospitals in Saudi Arabia? Ability, motivation, and opportunity (AMO) theory holds that if employees are competent, skilled, and motivated, then they can create more opportunities at the personal and organizational levels. Therefore, organizations should provide training and career advancement opportunities to employees, as this can enhance their skills and motivation and can drive organizational productivity.

There are various fields in which institutional theory has been applied, such as political science, economics, organizational theory, and sociology. However, there is little understanding about the application of institutional theory in public hospital settings. In the context of institutional theory, organizations embed themselves within different environments in which they may acquire legitimacy from imitative, coercive, or other kinds of institutional forces. Moreover, institutional theory implies that corporate decisions are partially driven by rational 
effectiveness goals and partially by legitimacy concerns and cultural and social factors. Companies adhere to different institutional values or norms to acquire a fit and/or to integrate into the surrounding environment. Isomorphism provides an in-depth understanding of the institutional environment. By incorporating institutional rules within their own structures, organizations become more homogeneous and similar in structure over time. This is particularly the case within particular institutional environments and contexts, such as how public hospitals in Arab countries, such as Saudi Arabia, manage SM use.

The 3 common methods of isomorphism identified by DiMaggio and Powell are coercive isomorphism (firms are either compelled to adhere to rules or adopt to structures), normative isomorphism (firms adopt forms as professionals within the firm who claim their superiority over others), and mimetic isomorphism (one firm copies another firm, often because of uncertainty). Institutionalization is a process that involves modifying behaviors to fulfill social expectations that are derived from different norms such as regulatory structures, professions, laws, cultural practices, and government agencies. All these actors exert pressure on firms to operate in a particular manner. Institutional theory is considered a powerful and popular explanatory tool for any analysis of organizational behavior that seeks to address different forms of organizational change. In contrast, the concept upon which most classical approaches are based is that organizations are dominated mainly by role, personal preferences, and the interest of individuals, as well as by rational actors. Likewise, new institutionalism primarily focuses on the formative role of organizations. In this sense, the core postulate is that rational actors within organizations always look out for their personal interests and exploit particular institutional limitations in pursuit of these. This view suggests that patterns of organizational actions are not shaped by instrumental calculations, but by institutional forces, such as cultural scripts and norms. The deep penetration of organizations in social environments could expedite or compromise their use of SM platforms for organizational productivity purposes (Figure 1). This suggests that organizational procedures and structures often reflect the environmental expectations that are required to enhance the use of SM to make the organization more successful. Institutional theory is based on the idea that organizations always want to respond to their environmental demands with the purpose of leveraging competitive advantage.

Figure 1. Theoretical framework based on institutional and ability, motivation, and opportunity theories.

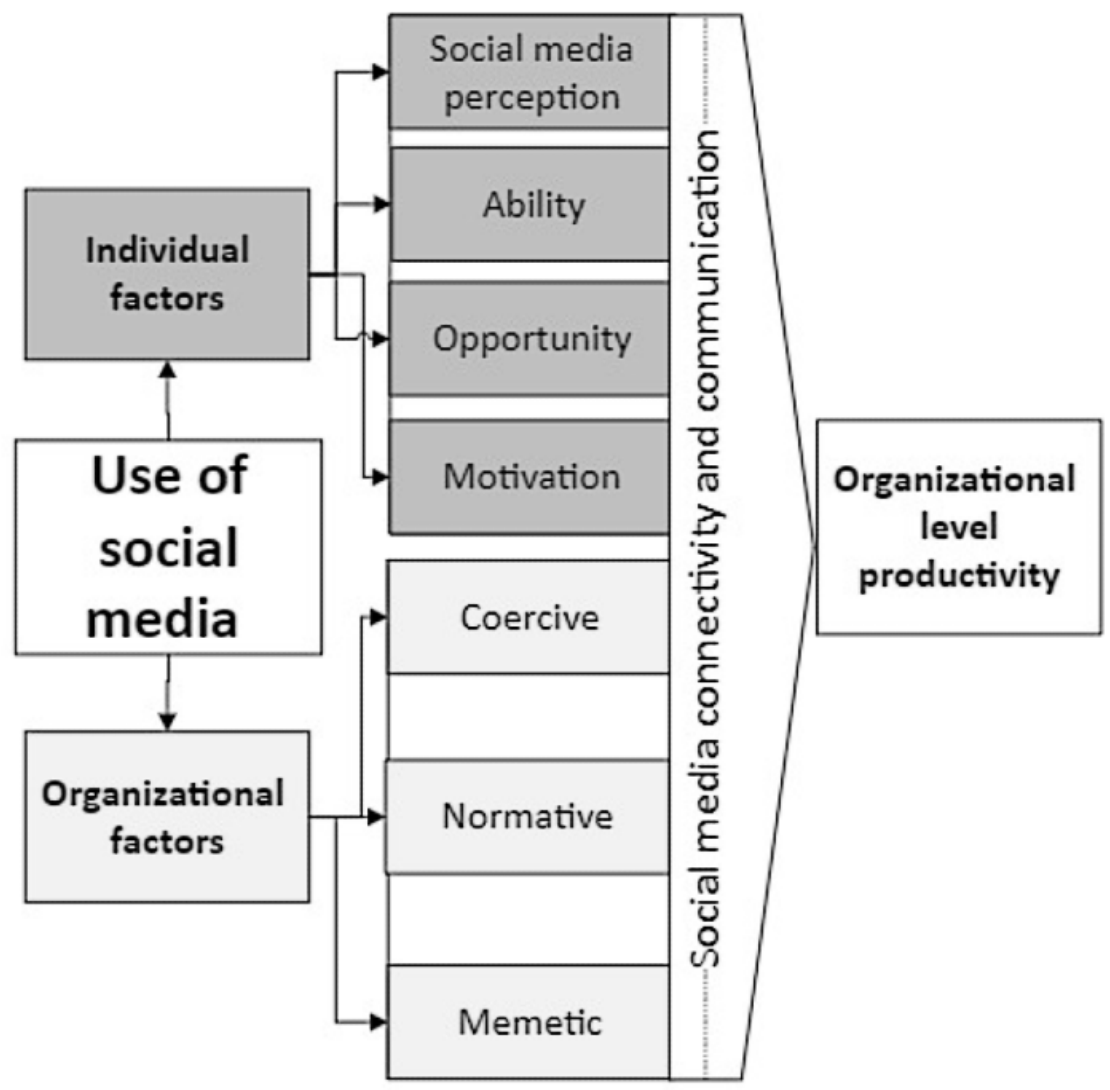

\section{Methods}

\section{Overview}

There is an increasing trend among researchers to use social constructivism to understand and synthesize the different realities of multiple disciplines, including the health sciences, philosophy, sociology, and psychology [1,21,22]. In particular, health science researchers extensively use social constructivism to gain in-depth insights into how health care professionals obtain and integrate new knowledge. They also tend to explore the kinds of behaviors they can develop to improve their own decision-making performance [22]. According to social constructivists, the social world in which we live has unique 
local, cultural, and social contexts, and all these contexts vary from location to location, from individual to individual, and from nation to nation [17,23]. Unique and different knowledge insights are, thus, produced through the lens of social constructivism because they critique shared beliefs, shared meaning, shared language, collective cultures, values, and social norms $[21,23]$. Social constructivists construct knowledge on the basis of cultural and social values. Therefore, they prefer to understand, see, and shape things in accordance with the viewpoints of social actors. The researchers were highly involved in this study to capture and better understand the issues under investigation. Institutional forces, national cultures, individual capabilities and abilities, social structures, organizational policies, the sharing and exchanging of knowledge, and SM are all subjective realities. Each of these influences the organizational productivity of public hospitals. Through the research process, this study clearly shows how social constructivism, analytical techniques, and data collection are interconnected.

There is limited literature available that has sought to uncover the influence of SM on employee work productivity in a public sector hospital. Therefore, this study is exploratory in nature because it aims to capture rich insights using social constructivism and interpretive methods. Social constructivists believe that there are multiple environmental and biological factors that have different impacts on individuals [17,21]. Interpretive methods are considered important where the researcher seeks to understand the social and real context of networking technologies and how social networking technologies influence organizational productivity in health care workplaces. Interpretive methods are thus recognized as important, especially when people are interested in knowing how the use of technology influences various institutional, government, cultural, and social structural factors [24,25]. Indeed, interpretive studies are more focused on understanding social contexts (ie, social relationships, peer influence, the division of labor, and the structure of an organization) that are impacted by the actions of individuals [17,21]. Previous studies have indicated that interpretive methods can capture subjective realties, such as individual levels of information and levels of knowledge, expectation, experience, and understanding [17,23]. Other studies have suggested that interpretive or qualitative methods are more useful for capturing the exploratory elements of subjects with the support of single or multiple theories [22]. These methods are usually useful for understanding individual perceptions about and sense-making in particular situations.

\section{Sampling and Population}

This study primarily focuses on Saudi public sector (government) hospitals, as they are strongly influenced by institutional, cultural, and social forces. Multiple hospitals were selected to gain insights from health care professionals in a variety of government hospitals in Jazan, Saudi Arabia. The hospitals selected for this study included Jizan Hospital, Prince Mohammed Bin Abdul Aziz Hospital, and King Saud Medical City. All these public sector hospitals have a rich technological and institutional history, and many health professionals want to work in them to gain valuable experience.
As many of the doctors in these hospitals are visiting professionals, it was not possible to fully describe the sampling framework. Moreover, although 2 of the hospitals were willing to provide a sampling framework, the others declined to provide any sampling framework. To address this issue, we decided to use a nonprobability sampling method. In health sciences, purposive sampling is common and is particularly useful when researchers are aware of the sampling framework and when they want to choose informants who have rich knowledge about the study objectives. Therefore, purposive sampling was used to collect data for this study. The 30 participants selected for data collection included nurses and head nurses working in government hospitals. Moreover, the 20 participants selected for focus group interviews included emergency doctors, surgeons, and hospitalists. Multimedia Appendices 1 and 2 indicate the demographic attributes of the selected informants. Their selection was based on the following inclusion criteria:

- Working within public sector hospitals for at least two years

- Older than 18 years

- Willing to voluntarily provide research data

- Had at least one active account on SM

\section{Data Collection Technique}

To collect qualitative data, 2 distinct forms of data collection techniques were used: focus group interviews and semistructured interviews. Data collection took about 7 months, and 3 different methods were used to gain in-depth insights into the expectations and experiences of professionals who use SM in the workplace. Semistructured interviews were undertaken because this type of method ensures the maximum participation of respondents throughout the research process. Moreover, "researchers have realized [for] quite some time that researchers are not invisible neutral entities; rather they are part of the interactions they seek to study and influence as well as observe those interactions." Of real interest here were the experiences of nurses and their perceptions of the different realities of organizational productivity and SM use. Doctors were seen as key sources for verifying and conceptualizing these shared realities.

Interpretative methods are useful for gaining rich insights through observations, semistructured interviews, stories, diaries, and narratives $[24,25]$. Semistructured interviews are one of the most commonly used interpretative methods among interpretivists because they can elicit rich insights and generate answers to multiple questions such as what, how, and why $[22,26]$. Moreover, the flexibility of semistructured interviews means that it is possible to add or modify the interview questions. Contrary to structured or open-ended interviews, semistructured interviews focused on obtaining particular answers $[24,25]$. Semistructured interviews were conducted in a face-to-face setting. The following questions were asked during semistructured interviews: How do you see the role of SM in relation to organizational productivity in the workplace? What socialization factors appear useful for increasing your productivity? What are your organizational and individual perceptions of SM use within hospitals? What challenges negatively influence SM use within this hospital? What are the specific public sector policies or factors that influence the use of SM in public sector hospital settings? 
Focus group interviews are widely used by researchers interested in accessing the opinions and life stories of individuals in specific situations. Many researchers recommend conducting focus group interviews whenever there is a need to handle discourse in which the image of the research participants is continuously regenerated. Health professionals were invited to participate in focus group interviews. The sample comprised 20 doctors and 30 nurses (Multimedia Appendices 1 and 2). This sample resonates with previous qualitative studies wherein the data saturation point was reached before the 30th interview $[24,25]$. To maintain social distancing, the social networks of health professionals and the researcher played an important role in identifying respondents with knowledge of SM technologies. Initially, the participants were asked if they would like to volunteer to participate in the interviews. In addition, some health professionals were contacted via SM (such as Twitter, Facebook, and WhatsApp). The researchers sent reminder emails to contact any participants who had not yet confirmed their interview schedule either by Skype or telephone. Interviews through Skype and telephone took place to maintain social distancing during the COVID-19 pandemic.

Interviews with nurses and head nurses lasted between 30 and 60 minutes. Skype videoconferencing calls were initiated to conduct focus group interviews with the selected health professionals. Participants preferred to take calls in their private offices in the hospitals in which they worked. Interview questions were developed based on the expectations and experiences of health professionals in relation to technology. This is an efficient way through which episodic memory can be accessed and complementary insights can be induced $[22,26]$. During interviews, episodic memory in individuals' neurocognitive memory systems can be activated by asking them about specific and ordered occasions [22,26], such as employee performance and SM, in this study. Moreover, the researcher used the past tense while developing interview questions to ensure the collection of complete data. Technical questions were also used in this study, whenever required. Many studies have recommended using tactical questions, particularly when managing participants seems quite difficult $[22,26]$. Indeed, tactical questions represent the summary of questions that were initially asked and are also very useful when it comes to addressing the risks identified earlier.

By asking different forms of interview questions, the researcher can optimize conversations with health professionals on the topic of internal generalizability [22]. Moreover, in the context of social constructivism, internal generalizability was possible with the help of data gathered from different respondents in terms of gender, occupation, and age [22]. The sample drawn for this study contained 30 female respondents of different ages (19-50 years) and experiences (Multimedia Appendix 1). The sample contained 20 doctors (Multimedia Appendix 2). Regardless of the sample composition (more males and fewer female participants), no screening questions preceded the interviews to avoid any intentional demographical influence on an otherwise diverse sample. The respondents were also provided with verbatim transcripts of the interviews to enable cross-examination, increase data validity, and improve internal generalizability.

\section{Thematic Analysis}

This study used thematic analysis to explore data and to develop a common set of themes or patterns. The researcher also used transcripts of the interviews to develop different initial codes to support the main themes of organizational networking and patient engagement. A total of 3 codes emerged around organizational networking: technological collaboration, policy conflict, and recorded data. In total, 4 codes of employee engagement emerged: socialization and trust, shared communication, ability and perception, and patient communication. After the construction of the main themes, keywords, and codes, the researchers reviewed them to increase both external and internal homogeneity [24,25]. Figure 2 illustrates the thematic analytical procedure of the Social Media Organizational Productivity (SMOP) model.

Data were organized into a verbatim transcript spanning 218 pages. The data were analyzed across 3 key phases. The responses of doctors, head nurses, and nurses were analyzed in the first phase of this process. Next, words that were repeatedly used throughout the interviews were identified. To group these repeated words into codes, the researcher visited them iteratively. Subsequently, the researcher assigned themes to these codes based on their meanings. Further analysis of responses was performed in the second phase. As a result, SM social practices are described. The integration of the experiences of doctors and head nurses was carried out in the third phase, so that a holistic outlook of organizational productivity and SM as a joint experience could be disclosed. 
Figure 2. Thematic analysis process.

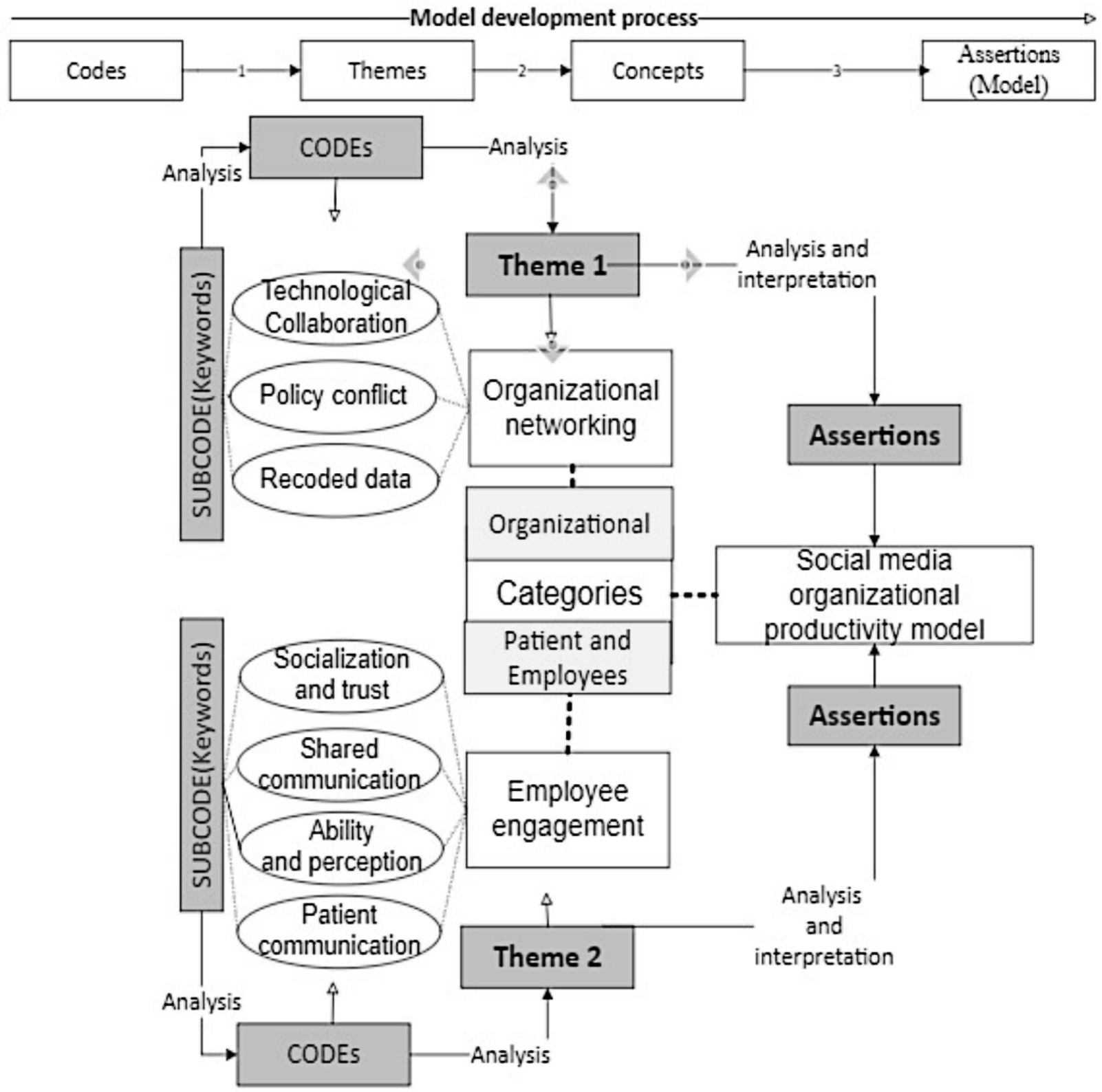

\section{Trustworthiness of Qualitative Findings}

Qualitative studies use confirmability, credibility, dependability, and transferability to maintain rigor and trustworthiness, whereas quantitative studies use reliability, objectivity, and validity to ensure rigor and trustworthiness. Internal validity should be replaced by credibility, external validity by transferability, reliability by dependability, and objectivity by confirmability. Although conducting qualitative studies, it is assumed that no truth is absolute in nature, but rather multiple realities are accessed. Consequently, generalizing the qualitative findings is not possible. However, credibility, confirmability, transferability, and dependability are, however, very useful measures of rigor and research validity for qualitative studies. Moreover, qualitative studies can improve the transferability of their findings by providing rich descriptions of the research process. This study, for example, produces transferable findings by highlighting the research process and providing a detailed justification for every qualitative data gathering method used. The extent to which the researchers or participants in qualitative studies can evaluate both the recommendations and interpretation of study findings is termed dependability. In this study, the researchers evaluated the data many times to ensure the rigor and trustworthiness of interpretations. Semistructured interviews were conducted to ensure the confirmability of the findings. The use of different qualitative data collection techniques with different respondents increased the credibility of the qualitative findings. In this study, the researcher ensured the credibility of the findings by using focus groups and semistructured interviews with a variety of participants. 


\section{Results}

\section{Main Theme 1: Organizational Networking}

Organizational networking involves exchanging, governing, and organizing sociotechnical networks that can enhance information exchange and social relationships within a formal organization [27]. This study of organizational networking provides ideas about how knowledge, information, communication, and decision making can be carried out in a formal setting. For example, Saudi public sector hospitals operate under the authority of the Ministry of Health. Therefore, these authorities can influence the level of knowledge, information, communication, and decision making, which ultimately influence the level of organizational productivity. To understand organizational networking in Saudi public sector hospitals, this study summarizes the findings into 3 codes: technological collaboration, policy conflict, and recorded data.

\section{Code 1: Technological Collaboration}

Technology collaboration refers to the systems and tools that help stakeholders to coordinate and complete tasks effectively, whether working remotely or in an office environment. It is found that nurses use SM instant messaging and video calls to increase interpersonal collaboration. As a result, they can enhance their knowledge, influence the speed at which they work, and increase their organizational productivity. For example, interviewee 19 noted:

social media increased peer to peer support as I can consult with my senior nurses through video call and messages and their shared knowledge is always helpful as now, I can be able to complete multiple tasks in limited time.

There is evidence that SM increases real-time communication, as video calls help health professionals manage patients in intensive care units (ICUs). Specialist doctors cannot be available at all times to deal with critical patients in ICUs. Therefore, SM provides them with the opportunity to manage situations through nurses so that they can assess the condition of multiple patients. For example, interviewee 22 noted:

I am working in ICU and many times I faced severe situation as I have to deal with emergency patients who have multiple diseases so we need sometimes urgent video call to different experts so they can advise what I should do until they physically visit to ICU.

There is evidence that SM has increased the virtual connectivity of different health professionals (ie, endocrinologists and pulmonologists), which means they are in a position to join group discussions with vulnerable patients. This could drive the need for emergency visits to assess vulnerable patients. For example, focus group interviewee 19 stated:

Social media helped to virtually connected with my patients as sometimes patients are more critical such as old (unable to visit hospitals) as well as having both diabetes and asthma at the same time so different medical experts (endocrinologist and pulmonologist) can now arrange video call for proper guidance of patients.

The COVID-19 pandemic has increased many risks to health professionals, patients, and communities. However, SM platforms such as WhatsApp and Skype help users to control situations, especially when there are limited health resources. Therefore, these SM platforms contribute to the care of COVID-19 patients. For example, focus group interviewee 5 noted:

Dealing with vulnerable patients has become more complicated, especially when they have the virus, so using Skype and WhatsApp video calls means we can coordinate with each other in a safe community.

\section{Code 2: Policy Conflict}

Policy conflict occurs when there is no professional code of conduct regarding the use of SM platforms. As a result, these social platforms cannot deliver maximum benefits at the organizational level. For example, interviewee 5 noted:

The Saudi ministry of health did not develop any organizational policy to use social media in workplace as a result we have not top management support otherwise we can practice it effectively to enhance the speed and collaborative care for patients.

There is evidence that although the use of SM helps professionals to collaborate and coordinate activities, there is no Saudi government policy to positively use these platforms in the workplace. Therefore, some health professionals use these social platforms, whereas others do not. For example, focus group interviewee 9 said:

I am working in public hospitals since 26 years but I experienced when we started to use social media then it saved our time, energy, financial resources, and use of physical infrastructure.

Some nurses felt that SM platforms really helped them to educate their patients so that they could control emergency situations and find the best way to deliver medicine and diet plans. Furthermore, many used SM to share relevant health care materials with patients, even in the absence of organizational support for the use of SM in public hospital settings. For example, interviewer 18 noted:

It is our duty to educate patient so that they can self-manage their diseases and reduce work overload and utilization of human and financial resources. Social media provided us this opportunity to share health care material with patients but there is no organizational policy and leadership support for using these best interactive communication platforms.

\section{Code 3: Recorded Data}

Recorded data refer to SM channels such as YouTube providing facilities to health professionals to record, store, and share data to save time, energy, and resources. In the past, health professionals have tended to verbally communicate to organize tasks that have no official record. As a result, many did not later accept responsibility if anything serious happened to the patients that were being discussed. For example, interviewer 2 noted: 
Social media helps to generate evidence of communication as, in the past verbal communication is not recorded and sometimes our seniors decline to take responsibility for their recommendations/actions.

Participants felt more engaged and connected with vulnerable patients and were able to manage emergency situations. As a result, they were able to deal with high numbers of patients, even when they were not at work. For example, focus group interviewee 19 highlighted:

I am a diabetes expert and sometimes I receive calls about my diabetes patients and how to clean wounds and stop bleeding in emergency situations...so social media really helps (me) to share YouTube videos of experts, so that these patients can self-manage in emergency situations.

Recording exchanges on SM also helped professionals to support parents and community education stakeholders by sharing knowledge about, for example, the care of infants among new mothers. For example, focus group interviewee 17 said:

I am working in the maternity department so we always share videos to educate the first-time parents about how they should lift their babies...the best positions for burping, and how they should use infant car seats to effectively care for their new-borns.

\section{Main Theme 2: Employee Engagement}

Employee engagement is an increasingly well-researched concept, and many have sought to understand this phenomenon through qualitative and quantitative methods. However, there is less understanding of how engaged health professionals and patients can influence organizational-level productivity, especially in the context of Saudi public sector hospitals. Employee engagement can be explained as motivated and enthusiastic employees involved in positive actions to improve health care services, reputations, and levels of organizational productivity. Although previous studies have provided an understanding of employee engagement from motivational, emotional, and cognitive perspectives, this study examines how SM can increase engagement among health professionals by building socialization and trust and by relying on shared communication, abilities and perceptions, and patient communication.

\section{Code 1: Socialization and Trust}

Socialization refers to the enhancement of information exchange to support learning and to improve competencies that can enhance productivity in hospitals. Socialization can promote effective communication among health professionals, and it is therefore useful for building trust in the workplace. For example, interviewee 23 noted:

\section{WeChat, Facebook, WhatsApp, and twitter are common social media platforms that we use to communicate and learn from doctors and colleagues. Therefore, we are not more effective and productive compared to the past.}

Socialization promotes the use of social networking platforms (ie, Twitter, WhatsApp, Facebook, and WeChat) for effective communication and is, therefore, an informal, popular, and optimal way of maintaining professional trust in intensive health care settings. The analysis suggests that doctors and nurses are now more connected and engaged and that SM is useful for celebrating individual and organizational achievements. These professionals are now more familiar with SM and can use it in emergency situations. For example, focus group interviewee 13 noted:

Nurses and doctors of a particular department are
connected through instant messaging apps for
celebration of new appointments, promotions,
personal educational achievements, parties, and
helping others during excessive work overload.

Some argue against the use of social networking platforms and believe that these have reduced productivity. For example, interviewee 18 noted:

I noticed many of my colleagues and seniors even using social media in ICU and operation theatres when they have to focus on their work, so I believe its wastage of time and reduce the work productivity.

\section{Code 2: Shared Communication}

Shared communication refers to explaining, questioning, listening, understanding, acting, advocating, and negotiating over tasks, roles, and responsibilities to enhance organizational productivity. For example, interviewee 13 said:

\section{During this global pandemic, both nurse and doctors are extremely busy in ICU and critical operations, but the use of social media increase their interaction, coordination and collaboration especially when health resources are become limited due to increasing the number of patients.}

It is evident that the use of SM has driven coordination and collaboration among doctors and nurses, and they are able to manage more tasks within a limited time frame. The findings also suggest that mental and behavioral issues are heightened among children and older people. Therefore, using SM, doctors and nurses can more effectively discuss health care plans with patients during a global pandemic. For example, focus group interviewee 3 noted:

\section{The global pandemic has increased the issues of children and old people who have behaviour and psychological issues. So, the use of social media facilitates shared communication so parents and other people may know what care should be more effective without visiting in hospitals.}

The findings suggest that multiple doctors now collaborate through SM to discuss health care plans with patients who have various diseases and who are vulnerable during global pandemics. For example, focus group interviewee 4 said:

I have some patients who have type 2 diabetes, cancer, asthma issues and they are older therefore it is not risk free to meet with them physically. Therefore, sometimes multiple doctors (Trauma surgeon, Endocrinologist, and Pulmonologist) 
conduct conference call for discussing the medication and health care plan.

\section{Code 3: Ability and Perception}

Ability refers to the particular skills that we elicit from sharing practical knowledge and experiences through SM platforms. The interviewees had mixed views, and some acknowledged how groups on SM updated their skills and performance, whereas others complained that SM platforms increased expectations when it came to response times. For example, interviewee 9 noted:

\section{Our hospital IT department is not very skilled, so we got lower motivational and technical support. Some of our colleagues also have perception that use of these social media platforms created more stress and pressure from our seniors who always demand quick response and action.}

However, focus group interviewee 14 said:

Our profession is required to continuously learn and improve our ability, so I have joined many YouTube channels and WhatsApp groups where we are getting opportunities to learn, knowledge exchange, and improve our performance.

SM has, therefore, connected professionals who have relevant, rich experiences and practical knowledge of the different workplaces at national and international levels. Therefore, there are opportunities for junior staff to increase their skills by learning through experience. For example, interviewee 14 noted:

\begin{abstract}
We (head nurses of different hospitals and countries) are now connected with WhatsApp and Facebook groups where we sharing content related to advance healthcare techniques, so we experienced that our level of performance is now more improved.
\end{abstract}

\section{Code 4: Patient Communication}

Keywords: patient communication, health care, high risks, education, awareness, skills, knowledge.

SM helps to create better health services and patient-centered care. Therefore, doctors are more engaged, particularly with patients who require immediate help to recover from critical situations (ie, pneumonia, chest infections, and asthma). For example, focus group interviewee 9 noted:

\section{Instant messaging apps have created direct communication (audio, video, text messaging) between doctors and patients. So now I can more effectively work on asthma patients regarding diet plan, smoking/alcohol habit, inhaler use, and how to control stress in emergency situations.}

In the presence of COVID-19, both public and private hospitals have insufficient resources to provide health care to every mother. Therefore, doctors often only engage with mothers who are struggling because of high-risk pregnancies. For instance, focus group interviewee 6 highlighted:

Due to the spread of Covid-19, we cannot ask every patient to come in hospital therefore those mothers who have high risks birth and pregnancies may only contact us through social media and telephone when they need caesareans and interventions.

The findings highlight that the global pandemic has increased the responsibilities of nurses and doctors who are now less able to provide high standard health care services. However, SM platforms provide them with an opportunity to disseminate educational materials about chronic diseases so that emergency visits can be reduced. For example, focus group interviewee 5 noted:

Every asthma patient has different level of complications, education, awareness, and knowledge so social media provided me more opportunity to share relevant material with those asthma patients who have low knowledge as they do more emergency visits and increase work overload during global pandemic.

\section{Discussion}

\section{Principal Findings}

The findings show that the increased use of SM has helped to enhance both networking and connections among health professionals outside and within public hospitals. Due to the advent and use of SM sites, management in public hospitals can more quickly share the medical histories of critical patients and can ask for advice from experts in other hospitals. SM promotes knowledge and information exchange among health professionals. As a result, they have become more knowledgeable and competent. There is evidence that various global environmental changes and the increasing consumption of petrol have increased the level of air pollution in Saudi Arabia. Therefore, the number of chronic diseases, especially the number of asthma patients, has increased in recent years. Furthermore, the number of patients with type 2 diabetes has increased because of the high use of red meat and junk foods. These chronic patients have lower levels of education and awareness about chronic diseases, including how to avoid them. Health professionals and the Ministry of Health in Saudi Arabia have started to use YouTube and Twitter platforms to educate patients with chronic diseases. This can reduce work overload, emergency visits, and resource utilization. YouTube videos educate patients about medication, healthy diets, the use of inhalers, exercise plans, and methods to inject insulin to control diabetes.

There is a need to further enhance the productive use of SM as it enhances collaboration and socialization among health professionals and patients. For example, when a health professional is linked to patients with type 2 diabetes through SM, a shared video on YouTube can provide fruitful advice about exercise, the use of medication, the use of inhalers, healthy diets, and other ways to control diabetes. For example, if nurses and doctors are engaged through SM, they can manage emergency situations better, especially during public health crises when all patients cannot receive the same level of care and attention from doctors. Therefore, SM use enhances the level of information sharing. It facilitates learning, knowledge exchange, and patient education about healthy lifestyles. Using SM, patient engagement can enhance the experiences and 
knowledge of health professionals, which is useful for improving individual and organizational performance. Furthermore, SM helps to educate and activate patients and can therefore reduce the workload of health organizations as patients are able to control critical situations. Within the organizations in question, the increasing use of SM among nurses and doctors is beneficial for multitasking, as health professionals can ask, guide, or instruct others on routine and emergency tasks. Therefore, it can be argued that the use of SM enhances information sharing, knowledge, skills, and time efficiency for health professionals who are now more productive as a result.

The analysis revealed that Saudi hospitals operate under the control of the Saudi Ministry of Health. Therefore, this ministry can create policies, rules, and regulations that can influence communication, decision making, and the level of productivity of health professionals. It is evident that the use of SM drives collaboration and coordination among health professionals and patients. However, unfortunately, there is no Saudi Health Ministry Policy to positively use these platforms in the workplace. Therefore, when health professionals use these platforms, there is no training or technical, motivational, or organizational support for them. This is counterintuitive, given that SM can save professionals' time, energy, and resources. The findings reveal that the use of SM enhances peer-to-peer support, as newly appointed nurses and young doctors can consult with head nurses and senior doctors through video calls. Their shared knowledge can help them, especially when patients are in emergency situations. However, analysis also suggests that the use of SM has created a culture of quick responses and actions among junior professionals, which ultimately increases the stress and pressure they face to fit more work into limited timeframes. Both nurses and doctors use instant messaging and video calls to increase interpersonal and professional collaboration. As a result, they are able to enhance their level of knowledge, speed of work, and overall organizational productivity. Therefore, it is recommended that the Saudi Ministry of Health and government should specify regulations and policies that can increase the use of SM platforms as evidence-based practices that help health professionals communicate and collaborate to manage critical patients.

A key finding of this study is that the use of SM has promoted the exchange of recorded data and technological collaboration among health professionals. This has improved patient-centric communication, health care services, and the overall level of productivity. For example, diabetes experts can now conduct conference calls with their diabetes patients and advise them on how to clean wounds and stop bleeding in emergency situations. YouTube videos of health experts meant that these patients could self-manage in emergency situations, especially when visiting a hospital is risky. These SM support technological collaboration among different health professionals when it comes to diabetes and asthma health experts (endocrinologists and pulmonologists) who can now arrange video calls to share best practices in terms of medication, diets, and health care plans. This is especially useful for patients with multiple diseases. There is evidence that nurses use SM to share videos to educate first-time parents about infant childcare. Indeed, the data sharing facility provided by SM saves nurses' time so that they can prioritize clinical care and balance it with educating parents and patients.

The use of SM enhances employee engagement by building socialization and trust. It supports shared communication and better patient communication, especially during a global pandemic when health resources are limited, and it is less common for doctors to see patients in person. The findings also reveal that both nurses and doctors engage with instant messaging apps to join appointments. They also use these to share information about promotions, personal educational achievements, parties, and mutual self-help. Therefore, socialization through SM increases social interactions, knowledge exchange, and trust building, and such media can help with emergency situations. Such shared communication is facilitated through SM as doctors and nurses can discuss health care plans and behavioral issues in relation to patients during global pandemics. During global pandemics, both nurses and doctors are unable to provide high standards of health care services because of limited human capital and health resources. However, SM platforms provide them with the opportunity to disseminate educational materials about chronic diseases so that emergency trips to hospitals can be reduced, whereas awareness and self-care can be increased.

\section{Contribution}

Previous researchers have applied various models and theories to understand the adoption of technology or SM in the workplace. Examples include the likelihood model [28,29], the technology acceptance model [30-32], the unified theory of acceptance and use of technology 2 [33], the diffusion of innovation theory [34], the social cognitive theory [15], the theory of planned behavior model [35], social exchange theory [3], and social influence theory [25]. However, none of these models have been tested when people are practicing social distancing and are restricted to their homes because of a pandemic. This is the first study to explore how SM practices are developed at the institutional level with the purpose of enhancing technological collaboration among health professionals and patient-centric approaches during the COVID-19 pandemic.

In terms of the first theoretical contribution, this study applied institutional and AMO theories to understand how health professionals experience instant messaging apps. The results could be used to improve professional socialization, trust building, data recording, knowledge exchange, collaboration, health service delivery, and organizational productivity. For example, institutional theory offers knowledge about how to use accessible technology to deal with uncertainty, whereas the ability and motivation of health professionals to use SM provides them with the opportunity to cope with uncertainty during a global pandemic. It empowers them to educate patients and, subsequently, lowers emergency visits.

The second theoretical contribution is the use of institutional theory through the lens of health professionals' motivations and opportunities that are explored in the specific context of Saudi public sector hospitals. For example, institutional theory offers an understanding of professional networking and collaboration, whereas the AMO theory provides an understanding of how 
both diabetes and asthma health experts (endocrinologists and pulmonologists) can arrange video calls to share best practices in terms of medication, diet, and health care plans for patients who have multiple diseases and who are vulnerable during a global pandemic. The third and most significant theoretical contribution is that the SMOP model was developed in this study. This model suggests that, even in the absence of institutional policies and in the context of uncertainty, the use of SM platforms at the individual level provides the motivation to develop health professionals and patient interactions with the purpose of creating health care plans during a global pandemic (Figure 3). This model provides an understanding of how to use SM to increase the accessibility of health care professionals at the institutional level. This way, shared communication can be used to manage patients who are at high risk (eg, older and children who live with psychological and behavioral issues).

Figure 3. Social Media Organizational Productivity model.

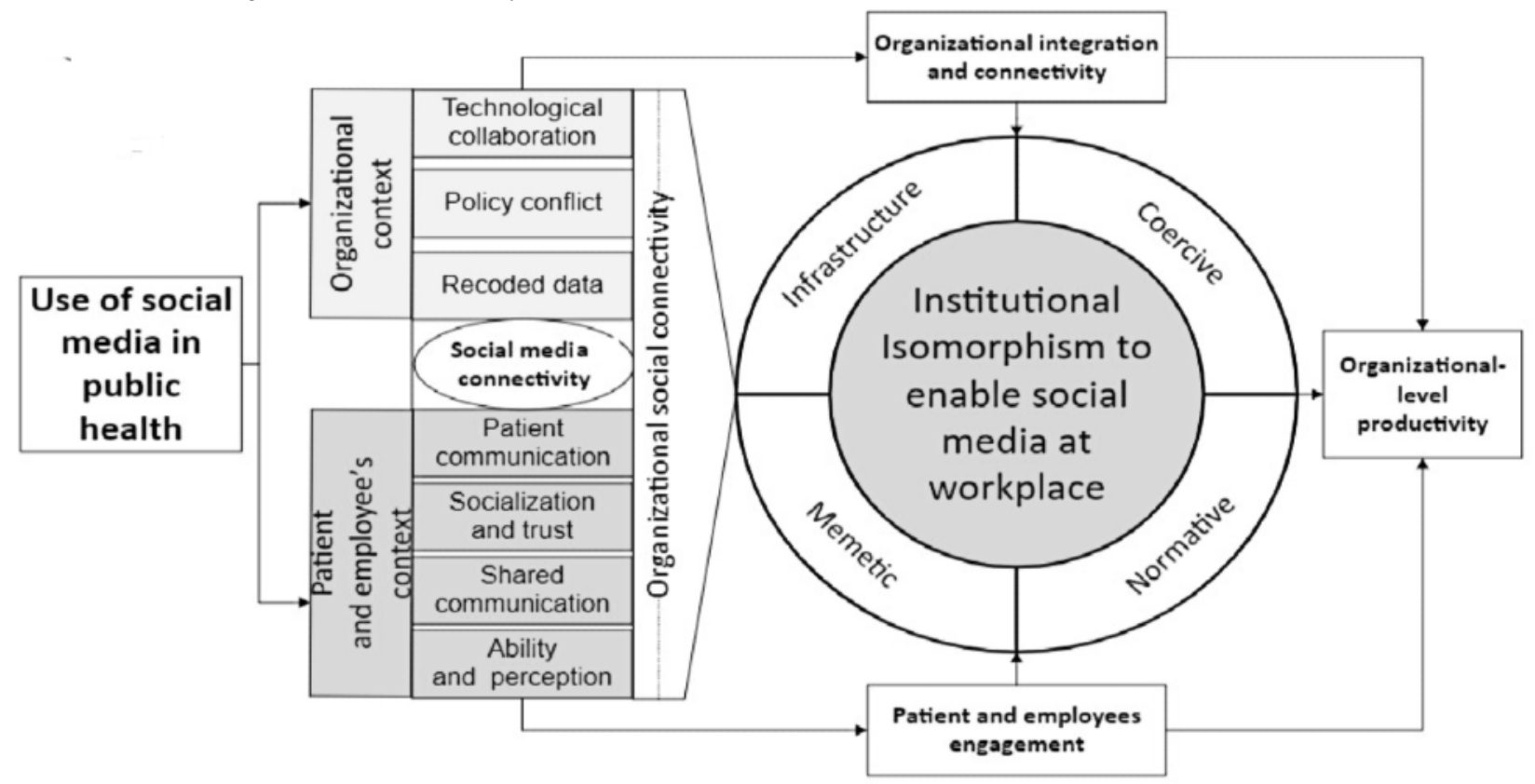

\section{Practical Implications}

This study has provided useful insights regarding how health professionals can make the best use of SM platforms to deal with uncertainty during global pandemics and can reduce the number of emergency visits patients make to hospitals. The second practical contribution is that the use of SM has created a means to record data on how, for example, new parents should care for newborns and how patients with asthma and patients with type 2 diabetes can manage emergency situations. SM can also decrease the workload of professionals during a global pandemic. These data and the technological collaboration between health professionals and patients can reduce the necessity for hospital admissions, and it is useful to ensure the safety of families, communities, and working environments during global pandemics. The third practical contribution is how the use of SM creates an opportunity, regardless of circumstances, to enhance the productive use of SM during a global pandemic. Therefore, it is recommended that the Saudi
Ministry of Health and Saudi government should create institutional policies that support the use of SM platforms in public and private hospital settings.

\section{Limitations and Future Directions}

Although this study provides unique theoretical and practical contributions, it is not free from limitations. For example, previous studies have highlighted that although qualitative methods can provide rich insights to develop theoretical frameworks, they cannot test the validity of theoretical frameworks [24,25]. Therefore, future studies may test the validity of the SMOP model by collecting data from health professionals in private and public hospitals using statistical methods. Future studies might also test the effectiveness of the SMOP model in different environments and contexts where there are institutional policies and skilled leadership teams that can create more motivation and opportunities to use SM platforms to increase organizational productivity.

\section{Conflicts of Interest}

None declared.

\section{Multimedia Appendix 1}

Demographic information of doctors. [DOCX File, 14 KB-Multimedia Appendix 1] 


\section{Multimedia Appendix 2}

Demographic information of nurses.

[DOCX File, 15 KB-Multimedia Appendix 2]

\section{References}

1. Galbin A. An introduction to social constructionism. Social Research Reports. 2014. URL: https://www.researchreports.ro/ an-introduction-to-social-constructionism [accessed 2021-04-09]

2. Saudi Arabia is the most Twitter-crazy country in the world: Business Insider. Ministry of Communication and information technology. URL: https://www.mcit.gov.sa/en/media-center/news/91426 [accessed 2020-10-22]

3. Chen X, Wei S. The impact of social media use for communication and social exchange relationship on employee performance. J Knowl Manag 2020 Jun 19;24(6):1289-1314. [doi: 10.1108/jkm-04-2019-0167]

4. Cheng T, Zhang P, Wen Y, Wang L. Social media use and employee innovative performance: work engagement as a mediator. Soc Behav Pers 2020 Jan 07;48(1):1-9. [doi: 10.2224/sbp.8698]

5. Benitez J, Ruiz L, Castillo A, Llorens J. How corporate social responsibility activities influence employer reputation: the role of social media capability. Decis Support Syst 2020 Feb;129:113223. [doi: 10.1016/j.dss.2019.113223]

6. Martinez-Millana A, Fernandez-Llatas C, Bilbao IB, Salcedo MT, Salcedo VT. Evaluating the social media performance of hospitals in Spain: a longitudinal and comparative study. J Med Internet Res 2017 May 23;19(5):e181 [FREE Full text] [doi: 10.2196/jmir.6763] [Medline: 28536091]

7. Van de Belt TH, Engelen LJ, Berben SA, Teerenstra S, Samsom M, Schoonhoven L. Internet and social media for health-related information and communication in health care: preferences of the Dutch general population. J Med Internet Res 2013 Oct 02;15(10):e220 [FREE Full text] [doi: 10.2196/jmir.2607] [Medline: 24088272]

8. Townsend A, Leese J, Adam P, McDonald M, Li LC, Kerr S, et al. eHealth, participatory medicine, and ethical care: a focus group study of patients' and health care providers' use of health-related internet information. J Med Internet Res 2015 Jun 22;17(6):e155 [FREE Full text] [doi: 10.2196/jmir.3792] [Medline: 26099267]

9. Antheunis ML, Tates K, Nieboer TE. Patients' and health professionals' use of social media in health care: motives, barriers and expectations. Patient Educ Couns 2013 Sep;92(3):426-431. [doi: 10.1016/j.pec.2013.06.020] [Medline: 23899831]

10. Keller B, Labrique A, Jain KM, Pekosz A, Levine O. Mind the gap: social media engagement by public health researchers. J Med Internet Res 2014 Jan 14;16(1):e8 [FREE Full text] [doi: 10.2196/jmir.2982] [Medline: 24425670]

11. Griffis HM, Kilaru AS, Werner RM, Asch DA, Hershey JC, Hill S, et al. Use of social media across US hospitals: descriptive analysis of adoption and utilization. J Med Internet Res 2014 Nov 27;16(11):e264 [FREE Full text] [doi: 10.2196/jmir.3758] [Medline: 25431831]

12. Why spy on Twitter? For Saudi Arabia, it's the Town Square. The New York Times. 2019. URL: $\underline{\text { https://www.nytimes.com/ }}$ 2019/11/07/world/middleeast/saudi-arabia-twitter-arrests.html [accessed 2019-11-07]

13. Saudi lifting ban on Skype, WhatsApp calls, but will monitor them. Reuters. 2017. URL: https://www.reuters.com/article/ us-saudi-telecoms-ban-idUSKCN1BW12L [accessed 2017-09-21]

14. Badea M. Social media and organizational communication. Procedia Soc Behav Sci 2014 Sep;149:70-75. [doi: 10.1016/j.sbspro.2014.08.192]

15. Cao X, Guo X, Vogel D, Zhang X. Exploring the influence of social media on employee work performance. Internet Res 2016. [doi: 10.1108/IntR-11-2014-0299]

16. Huang Y, Singh PV, Ghose A. A structural model of employee behavioral dynamics in enterprise social media. Manage Sci 2015 Dec;61(12):2825-2844. [doi: 10.1287/mnsc.2014.2125]

17. Dong JQ, Wu W. Business value of social media technologies: evidence from online user innovation communities. J Strateg Inf Syst 2015 Jun;24(2):113-127. [doi: 10.1016/j.jsis.2015.04.003]

18. Rueda L, Benitez J, Braojos J. From traditional education technologies to student satisfaction in management education: a theory of the role of social media applications. Inf Manag 2017 Dec;54(8):1059-1071. [doi: 10.1016/j.im.2017.06.002]

19. Zhou L, Zhang D, Yang CC, Wang Y. Harnessing social media for health information management. Electron Commer Res Appl 2018 Jan;27:139-151 [FREE Full text] [doi: 10.1016/j.elerap.2017.12.003] [Medline: 30147636]

20. Moorhead SA, Hazlett DE, Harrison L, Carroll JK, Irwin A, Hoving C. A new dimension of health care: systematic review of the uses, benefits, and limitations of social media for health communication. J Med Internet Res 2013 Apr 23;15(4):e85 [FREE Full text] [doi: 10.2196/jmir.1933] [Medline: 23615206]

21. Allen BJ. Social constructionism. In: Engaging Organizational Communication Theory \& Research: Multiple Perspectives. Thousand Oaks, California, United States: SAGE Publications; 2005:35.

22. Taylor B, Francis K. Qualitative Research in the Health Sciences : Methodologies, Methods and Processes. London: Routledge; 2013:1-312.

23. Lock A, Strong T. Social constructionism: sources and stirrings in theory and practice. Cambridge University Press 2010. [doi: 10.1017/CBO9780511815454]

24. Aslam U, Muqadas F, Imran MK, Saboor A. Emerging organizational parameters and their roles in implementation of organizational change. J Organ Chang Manag 2018 Aug 13;31(5):1084-1104. [doi: $10.1108 /$ jocm-08-2017-0300] 
25. Naeem M. Developing the antecedents of social influence for internet banking adoption through social networking platforms: evidence from conventional and Islamic banks. Asia Pacific J Mark Logist 2020 Apr 23;33(1):185-204. [doi: 10.1108/apjml-07-2019-0467]

26. Thomas A, Menon A, Boruff J, Rodriguez AM, Ahmed S. Applications of social constructivist learning theories in knowledge translation for healthcare professionals: a scoping review. Implement Sci 2014 May 06;9(1):54 [FREE Full text] [doi: 10.1186/1748-5908-9-54] [Medline: 24885925]

27. Thornton SC, Henneberg SC, Naudé P. Understanding types of organizational networking behaviors in the UK manufacturing sector. Ind Mark Manag 2013 Oct;42(7):1154-1166. [doi: 10.1016/j.indmarman.2013.06.005]

28. Li CY. Persuasive messages on information system acceptance: a theoretical extension of elaboration likelihood model and social influence theory. Comput Hum Behav 2013 Jan;29(1):264-275. [doi: 10.1016/j.chb.2012.09.003]

29. Ngai EW, Tao SS, Moon KK. Social media research: theories, constructs, and conceptual frameworks. J Inf Manag 2015 Feb;35(1):33-44. [doi: 10.1016/j.ijinfomgt.2014.09.004]

30. Al-Maatouk Q, Othman MS, Aldraiweesh A, Alturki U, Al-Rahmi WM, Aljeraiwi AA. Task-technology fit and technology acceptance model application to structure and evaluate the adoption of social media in academia. IEEE Access 2020;8:78427-78440. [doi: 10.1109/access.2020.2990420]

31. Park N, Rhoads M, Hou J, Lee KM. Understanding the acceptance of teleconferencing systems among employees: an extension of the technology acceptance model. Comput Hum Behav 2014 Oct;39:118-127. [doi: 10.1016/j.chb.2014.05.048]

32. Wamba SF, Bhattacharya M, Trinchera L, Ngai EW. Role of intrinsic and extrinsic factors in user social media acceptance within workspace: assessing unobserved heterogeneity. Int J Inf Manag 2017 Apr;37(2):1-13. [doi: 10.1016/j.ijinfomgt.2016.11.004]

33. Ouirdi ME, Ouirdi AE, Segers J, Pais I. Technology adoption in employee recruitment: the case of social media in Central and Eastern Europe. Comput Hum Behav 2016 Apr;57:240-249. [doi: 10.1016/j.chb.2015.12.043]

34. Khan NA, Khan AN. What followers are saying about transformational leaders fostering employee innovation via organisational learning, knowledge sharing and social media use in public organisations? Gov Inf Q 2019 Oct;36(4):101391. [doi: $10.1016 /$ j.giq.2019.07.003]

35. van Zoonen W, Verhoeven JW, Elving WJ. Understanding work-related social media use: an extension of theory of planned behavior. Int J Manag Econo Soc Sci 2014;3(4):164 [FREE Full text]

\title{
Abbreviations
}

AMO: ability, motivation, and opportunity theory

ICU: intensive care unit

SM: social media

SMOP: Social Media Organizational Productivity

\author{
Edited by G Eysenbach; submitted 23.08.20; peer-reviewed by U Aslam, F Muqadas; comments to author 11.09.20; revised version \\ received 25.02.21; accepted 03.03.21; published 05.05.21 \\ Please cite as: \\ Dailah $\mathrm{Sr} H G$, Naeem $M$ \\ A Social Media Organizational Productivity Model: Insights From Public Health Professionals \\ J Med Internet Res 2021;23(5):e23792 \\ URL: https://www.jmir.org/2021/5/e23792 \\ doi: $10.2196 / 23792$ \\ PMID:
}

CHamad Ghaleb Dailah Sr, Muhammad Naeem. Originally published in the Journal of Medical Internet Research (https://www.jmir.org), 05.05.2021. This is an open-access article distributed under the terms of the Creative Commons Attribution License (https://creativecommons.org/licenses/by/4.0/), which permits unrestricted use, distribution, and reproduction in any medium, provided the original work, first published in the Journal of Medical Internet Research, is properly cited. The complete bibliographic information, a link to the original publication on https://www.jmir.org/, as well as this copyright and license information must be included. 